\title{
Prevention of Implant-Associated Infection in Neuromodulation: Review of the Literature and Prototype of a Novel Protective Implant Coating
}

\author{
Nestor D. Tomycz,, Christopher Payne, ${ }^{1}$ Boyle C. Cheng,, Luisa Hiller, ${ }^{1}$ \\ Rory Eutsey, ${ }^{2}$ Donald M. Whiting, ${ }^{1}$ and Saadyah Averick ${ }^{1}$
}

\begin{abstract}
Background: Implanting hardware into surgical sites increases the rate of infection associated with these sites. Without novel efforts to reduce this rate of infection, we can expect to see an increase in the number of hardware-associated infections as more patients are implanted with these devices. These infections often necessitate the removal of these devices resulting in a significant financial and clinical burden to patients. We developed a prototype antibiotic coating using products that are both low cost and that can be sourced easily. Our study aims to test the effectiveness of this coating against bacteria commonly observed in hospitalassociated infections.

Methods: The antibiotic coating was prepared by combining one gram of vancomycin and $500 \mathrm{mg}$ of ciprofloxacin in $50 \mathrm{~mL}$ of glycerol. The coating was examined for inhibition of growth of Pseudomonas aeruginosa PA14 and Staphylococcus aureus AH2486 and compared with the bacterial growth of the above bacteria in glycerol alone. The growth curves were plotted measuring the bacterial growth at $5 \mathrm{~h}$ intervals.

Results: The results of the growth curves clearly demonstrate a lack of bacterial growth when these bacteria are combined with glycerol combined with our selected antibiotic agents.

Conclusion: There appears to be a limited interest from device companies in developing new strategies for infection prevention associated with neurosurgical hardware, and we propose that this prototype will be an effective and low-cost solution to a large problem.
\end{abstract}

Keywords: neurosurgery; surgical implant infection; surgical site infection

D ESPITE THE INCREASING TREND for all surgeons to implant foreign materials, devices, and prostheses in the human body to manage disease, there has been a conspicuous lag in technologic innovation to protect such implants from infection. Implant-associated infections continue to engender enormous morbidity and cost because, despite a small number of case reports, infection usually heralds the demise of the implant. The majority of implant-associated infections necessitate complete or partial explant of foreign material as well as prolonged intravenous or oral antibiotic therapy.

Because of a dearth of innovation in the pharmaceutical and device industry, many surgeons have created their own strategies or rituals to reduce implant infections. Neuromodulation devices such as deep brain stimulators and intrathecal pumps are particularly vulnerable implants because of multiple material compositions and large surface areas. Innovations to protect such complex implants from infection would likely be generalizable to other implants in medicine.

As more companies enter the neurosurgical market with devices to implant within the nervous system, the problem of implant-associated infections in neurosurgery is likely to rise. Here we review the literature on strategies to prevent implantassociated infections in neurosurgery and describe our prototype and continuing design of a novel anti-biofilm coating.

\footnotetext{
${ }^{1}$ Neuroscience Institute, Allegheny General Hospital, Pittsburgh Pennsylvania, USA.

${ }^{2}$ Department of Biological Sciences, Carnegie Mellon University, Pittsburgh, Pennsylvania, USA.
} 
All surgical procedures, by virtue of necessitating suture material for surgical site closure, can be said to involve the implantation of foreign material. Implanted foreign materials increase the risk of surgical site infection (SSI) [1,2]. Biofilms are thought to be the reason that implant-associated SSI carries such high morbidity and usually necessitates explantation of the implant. Once bacteria adhere to an implant and excrete an extra-cellular matrix that shields them within a biofilm, they are significantly less susceptible to antibiotic and antiseptic agents. Biofilms also protect bacteria from the immune system of the host [3].

All implants in neurosurgery are not at equal risk of infection, and the risk of implant-associated SSI likely is related not only to implant characteristics themselves but also the site of implantation. Moreover, not all implants necessitate explantation in the setting of infection. For example, titanium instrumentation for spine fusion is one of the most common implants performed in neurosurgical operations. Most treatment paradigms, however, now recommend preservation of titanium instrumentation in the setting of infection [4].

Neuromodulation implants such as deep brain stimulators, spinal cord stimulators, vagal nerve stimulators, and intrathecal pumps are often extremely difficult to salvage in the setting of infection. The reasons for this are likely multifactorial but include the large surface area of these implants, their different material components, the healing potential of the patient population that obtains such implants, the location of the implant, and length of operation required to place these sophisticated nervous system-computer implants.

Biofilms are thought to be a major reason that implants in medicine necessitate explant in the setting of infection, and there has been little innovation to protect implants in neurosurgical procedures and prevent biofilm formation. There is often great reluctance for patients undergoing life-altering implants to undergo explantation of neuromodulation devices, and this often leads to surgical and medical salvage attempts that carry a high failure rate and add to the cost of neuromodulation SSI. In a recent study, the mean cost for a patient undergoing salvage explantation, and reimplantation operation for deep brain stimulation (DBS) infection was $\$ 75,505$ [5].

The global neuromodulation market that involves complex implants that interface nerves, spinal cord, and brain is expected to rise to about 6 billion dollars by 2020 [6]. As a result, there is likely to be a concomitant rise in the incidence of neuromodulation SSI in the coming years. There is therefore an urgent need for novel strategies to protect the burgeoning number of implants that will be implanted to modulate the human nervous system.

\section{Strategies for Infection Prevention in Neuromodulation}

There have been numerous, mostly surgeon-devised, strategies published to reduce SSI in neuromodulation. Although much of the data is limited by its retrospective and non-randomized design, the success of some of these strategies has been quite impressive. Halpern et al. [7] reported that self-administered pre-operative alcohol-based antiseptic wash reduced DBS infection from $9.02 \%$ to $0 \%$ in their center. Miller et al. [8] demonstrate that local neomycin/polymyxin antibiotic significantly reduced infections when applied locally in the surgical sites of patients undergoing stereotactic and functional neurosurgical hardware operations.

Recently, some have pointed out that despite decades of sporadic use by certain neurosurgeons, the application of topical antibiotic agents has been poorly studied in neurosurgery with only low-level class III evidence available [9]. One cannot say that class III evidence has no merit, but widespread use and acceptance will only likely be achieved if success is mirrored in randomized controlled studies.

One topical antibiotic strategy that has seen burgeoning usage across surgical specialities is intra-surgical site vancomycin powder. Although most data remain class III evidence, there is one randomized controlled trial of intra-surgical site vancomycin powder that showed benefit in spinal operation, and meta-analysis of 18 articles on the topic have confirmed a statistically significant reduction in SSI [10]. One immediately attractive aspect of vancomycin is its low cost, because a one gram vial at most institutions costs between $\$ 2$ and $\$ 12$ [11].

Despite its considerable success and safety record in spinal surgical procedures, there is limited data for intra-surgical site topical vancomycin in cranial neurosurgical operations and neuromodulation [12]. In neuromodulation, powdered vancomycin has shown some preliminary efficacy in reducing infections with paddle lead spinal cord stimulator and DBS implants [13,14]. Larger prospective studies are needed to determine further the safety and efficacy of implant protection with topical vancomycin in neuromodulation. Potential drawbacks of the simple topical vancomycin strategy are the development of resistance and failure to cover gramnegative organisms [15].

Although most neuromodulation SSI prevention strategies have been spearheaded by surgeons, there has been some implant infection prevention technology recently designed by industry. The most notable one is Tyrx (Medtronic, Inc), which has manufactured a biodegradable polyarylate mesh pouch that elutes two antibiotic agents-minocycline and rifampin - into the surrounding tissue. Implants such as pacemakers are placed inside the pouch, which degrades in a linear fashion over nine weeks.

Published studies have supported the use of Tyrx antibiotic mesh pouches for cardiac implantable electronic devices $[16,17]$. Approval from the Food and Drug Administration (FDA) has been obtained for neuromodulation implants such as the implantable pulse generators (IPGs) or batteries for spinal cord stimulation or DBS but currently there are no published data that support the use of Tyrx in neurosurgery. The high cost of Tyrx (about $\$ 800$ per pouch) has been a limitation for greater use and approval by hospital group purchasing organizations.

\section{Hardware Salvage in Neuromodulation SSI}

It is often impossible to salvage a neuromodulation implant in the setting of SSI, and this may be best exemplified by the fact that successful attempts are published in the literature. Certain implants such as baclofen pumps are particularly problematic to explant in the setting of SSI because baclofen withdrawal may be life-threatening. Thus, there is a literature of anecdotal case-report salvage strategies. Infected intra-thecal pumps have been saved in situ by the use of 
gentamicin-impregnated collagen fleece [18], rectus abdominis muscle flap [19], intra-reservoir teicoplanin, vancomycin, or gentamicin installation [20-23], intravenous linezolid [24]. Another baclofen intra-thecal pump and catheter were salvaged in the setting of bacterial meningitis by continuous coadministration of intra-thecal baclofen and vancomycin through the pump [25].

Finally, because complete explant of a neuromodulation system is often necessary, even successful partial hardware salvage has been reported in the literature. One case report describes salvage of the pump but partial removal of an intrathecal catheter in a patient with cancer pain; however, an irrigation and debridement surgical procedure was necessary in addition to intravenous antibiotic agents [26].

Although small series, these cases are notable given that studies of treating deep intra-thecal pump infections with intravenous antibiotic agents alone have reported a dismal $100 \%$ failure rate with all pumps ultimately necessitating explant to eradicate the infection [27]. The futility of hardware salvage in the setting of neuromodulation SSI is likely propagated through neurosurgical and infectious disease training and often becomes a self-fulfilling prophecy because of reluctance to attempt hardware salvage. While prevention of implant-related SSI remains paramount, there will remain a need for neurosurgeons and infectious disease specialists to develop evidence-based strategies for hardware salvage.

\section{Introduction of First-Generation Prototype Coating Technology in Neuromodulation}

An ideal implant-associated SSI infection prevention strategy would likely directly protect the implant from biofilm formation. In addition to safety and bioavailability, the protection strategy would be able to provide a barrier across the entire complex surface area of an implant and could be applicable to multiple different types of implants. Although custom, infection protection manufacturing has been applied to some orthopedic implants such as total joints manufactured with galvanic silver, a good strategy would could be quickly and safely applied post-manufacturing by a surgeon in the operating room before placing the implant within the patient.

Strategies such as vancomycin topical powder and antibiotic eluting meshes may increase the zone of protection around an implant but do not directly adhere or coat the threedimensional surface area of an implant where biofilms can form and force eventual explant. In addition, the implant coating strategy must be cost-effective to be applied universally in neuromodulation cases. Implant-associated infections may be increased by certain risk factors such as diabetes mellitus [28]; however, every patient undergoing a neuromodulation implant surgical procedure is at risk for infection. Thus, rather than applying infection-prevention strategies to a select group of high-risk patients, it seems more prudent to apply a low-cost strategy to all patients undergoing neuromodulation implant operation.

Our strategy was to develop a low-cost, bioavailable, and easy-to-prepare coating that could be applied rapidly or "painted" onto various neuromodulation implants such as intra-thecal pumps, DBS burr hole caps, and IPGs. The simple trihydroxy alcohol glycerin or glycerol was chosen as the base for multiple reasons. For one, glycerin is odorless, colorless, biodegradable, and non-toxic, and it has been recognized as a versatile and "green" solvent [29]. Glycerin does not require special handling or storage, and its safety in neurosurgical procedures has been well-established via its use in glycerol rhizotomy for trigeminal neuralgia [30].

In addition to its well-recognized safety and bioavailability, glycerin was attractive as the base for our coating because of its high viscosity and "stickiness," which made it seem appropriate to adhere and coat the large surface areas of neuromodulation implants. Moreover, glycerin is chemically inert and is recognized as safe by the FDA and Environmental Protection Agency. Glycerin is a common additive to food products and cosmetics, and no studies have shown that glycerin has any teratogenic or genotoxic effects.

We chose a dual-antibiotic strategy with vancomycin and ciprofloxacin to have broad-spectrum coverage of both grampositive and gram-negative organisms that may cause neuromodulation implant-associated infections. The safety and effectiveness of local vancomycin application has already been discussed in neurosurgical procedures. Ciprofloxacin has broad-spectrum activity against both gram-negative and gram-positive organisms and is FDA approved for ophthalmic use [30]. Moreover, ciprofloxacin has shown the ability to inhibit the growth of both Pseudomonas aeruginosa and Staphylococcus aureus when loaded into keratose hydrogels [31]. Bacteria resistant to both vancomycin and ciprofloxacin are not common, and this combination of antibiotic agents may exhibit synergistic killing toward certain strains of bacteria [32].

\section{Testing our Prototype Coating}

We prepared a broad coverage antibiotic coating by combining one gram of vancomycin and $500 \mathrm{mg}$ of ciprofloxacin in $50 \mathrm{~mL}$ of glycerin (all materials United States Pharmacopeia grade). Materials were combined by manually mixing powdered antibiotic agents into glycerin for $5 \mathrm{~min}$. The resultant suspension was examined for inhibition of growth within planktonic bacterial cultures. To test the growth of $P$. aeruginosa PA14 and $S$. aureus AH2486, we performed standard growth curves in planktonic cultures. Cultures were grown to $\log$ phase (OD600 of $\sim 1$ ) in tryptic soy broth (TSB) shaking at $37^{\circ} \mathrm{C}$. These were then diluted back to an OD600 of 0.05 in TSB with 60-70 mg of antibiotic glycerol, in TSB with glycerol alone, or with TSB only. The OD600 was then measured once per hour for $5 \mathrm{~h}$. This study did not require ethical approval or patient consent.

Our results clearly demonstrated a lack of bacterial growth when these bacteria are combined with glycerol combined with our selected antibiotic agents (Fig. 1). In addition, we have tested our glycerin-dual antibiotic coating with additional dye additives to improve intra-operative visual confirmation of the degree of implant coating. The colorless nature of glycerin and our coating can make it difficult to determine visually whether the complex surface area of an implant has been uniformly and fully coated. Our prototype coating was combined with three different dyes-fluorescein, methylene blue, and indacyanine green-and applied to a neuromodulation implant to improve the intra-operative visibility of the coating (Fig. 2).

Despite the significant clinical problem of implantassociated infections in medicine, the high sample size and 


\section{A P. aeruginosa antibiotic glycerol growth}

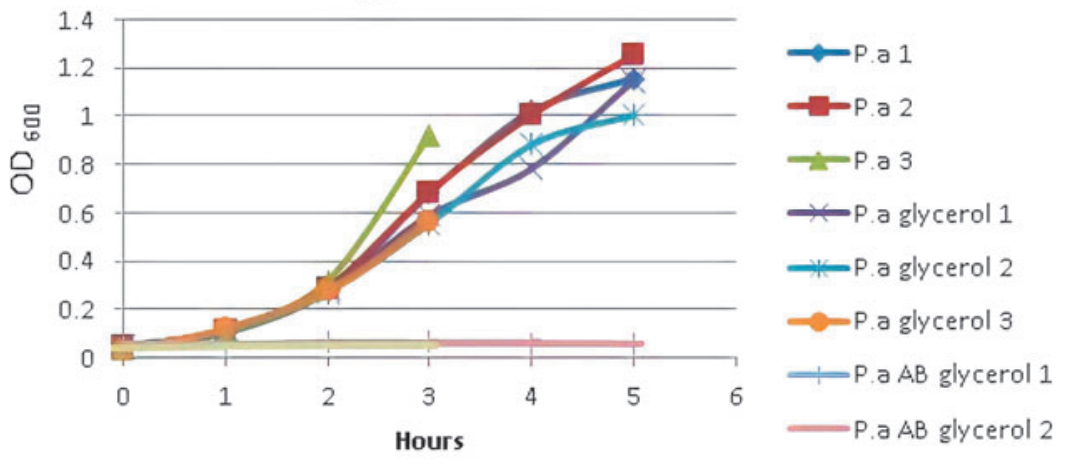

\section{B S. aureus antibiotic glycerol growth}

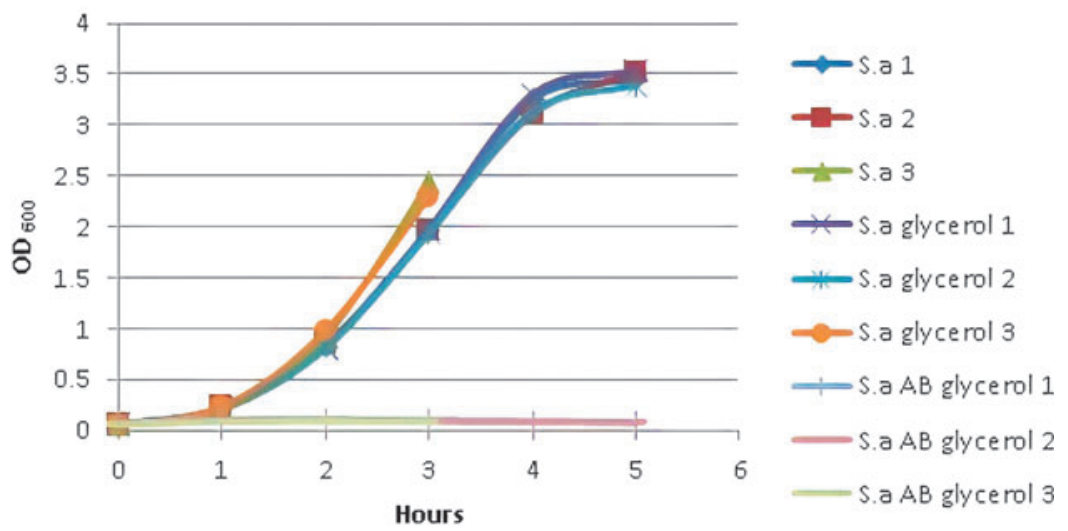

FIG. 1. Cultures grown to log phase (OD600 of 1) in Tryptic Soy Broth (TSB) shaking at $37^{\circ} \mathrm{C}$ and diluted back to an OD600 of 0.05 in TSB with $60-70 \mathrm{mg}$ of antibiotic glycerol, TSB with glycerol alone, or TSB only. The OD600 was measured once per hour for $5 \mathrm{~h}$. Inhibition of bacterial growth with glycerin combined with antibiotic agents is shown with (A) Pseudomonas aeruginosa PA14 and (B) Staphylococcus aureus AH2486. Color image is available online.
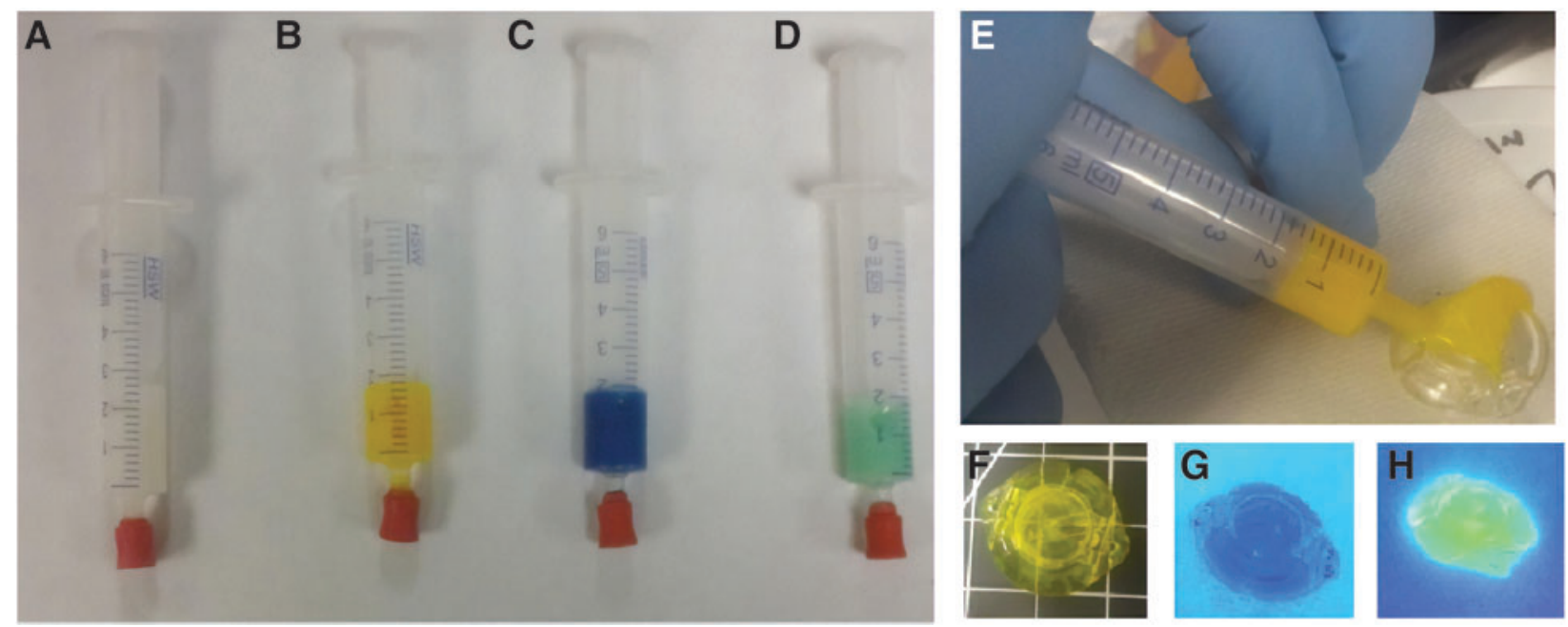

FIG. 2. Shows syringes filled with the antimicrobial and glycerin solution without dye (A), with $50 \mu \mathrm{L}$ of fluorescein $(100 \mathrm{mg} / \mathrm{mL}$ in water) (B), with $50 \mu \mathrm{L}$ methylene blue $(10 \mathrm{mg} / \mathrm{mL}$ in water) $(\mathbf{C})$, and with $50 \mu \mathrm{L}$ of indocyanine green $(2.5 \mathrm{mg} / \mathrm{mL}$ in water) (D). The ease of application to a deep brain stimulator burr-hole cover is demonstrated (E) as well as how these caps would appear under white light $(\mathbf{F})$ as well as under black light $(\mathbf{G}, \mathbf{H})$. Color image is available online. 
resultant costs of infection prevention studies in surgical procedures make it unlikely that any industry will perform randomized studies of infection prevention with coating technologies in the near future. Nevertheless, we have designed a cheap, simple, and accessible first generation coating that we hope will attract industry partners to further improve and market such infection prevention technology in neuromodulation and other fields of medicine. We are currently collaborating with one company to improve the delivery system for our coating technology, and further studies are under way with plans to examine the outcome of using this coating on hardware before implantation in patients.

\section{Conclusions}

Surgical implants have demonstrated their potential to significantly improve human health and longevity, and neuromodulation devices, which interface with the human nervous system, have become one of the fastest growing markets in the world. The sophistication of implants has accelerated, and "smart," closed-loop, brain-machine devices with the ability to treat patients with common neurologic diseases such as epilepsy and Alzheimer disease are rapidly approaching the clinical arena. Nevertheless, the age-old problem of microbes remains, and bacteria remain the number one enemy of surgical implants.

Most implant-associated infections necessitate hardware explantation, which carries significant cost and morbidity, and despite the scope of this problem, there has been little innovation to prevent implant-associated infections. Moreover, it is well-recognized that demonstrating infection prevention with any intervention necessitates significant expenditure and very large sample sizes, which will likely be a deterrent to randomized studies of protective surgical coatings in the near future.

That necessity is the mother of invention is well exemplified by the recent prevalence of local surgical site application of vancomycin by surgeons across multiple specialties. As medicine transitions to improved quality metrics, infections are becoming less acceptable, and novel strategies to prevent implant-associated infections are greatly needed. In the absence of commercially available and affordable products, surgeons should be encouraged to develop their own strategies for infection prophylaxis, and we hope that industry will follow suit.

\section{Acknowledgments}

The authors would like to thank the Department of Biological Sciences at Carnegie Mellon University for their assistance with this work.

\section{Author Disclosure Statement}

No competing financial interests exist.

\section{References}

1. Osterberg B, Blomstedt B. Effect of suture materials on bacterial survival in infection wounds. An experimental study. Acta Chir Scand 1979;145:431-434.

2. Leaper D, McBain AJ, Kramer A, et al.. Healthcare associated infection: Novel strategies and antimicrobial im- plants to prevent surgical site infection. Ann R Coll Surg Engl 2010;92:453-458.

3. Leid JG, Willson CJ, Shirtliff ME, et al. The exopolysaccharide alginate protects Pseudomonas aeruginosa biofilm bacteria from IFN-gamma-mediated macrophage killing. J Immunol 2005;175:7512-7518.

4. Kasliwal MK, Tan LA, Traynelis VC. Infection with spinal instrumentation: Review of pathogenesis, diagnosis, prevention, and management. Surg Neurol Int 2013;4(Suppl 5):S392-S403.

5. Chen T, Mirzadeh Z, Lambert M, et al. Cost of deep brain stimulation infection resulting in explantation. Stereotact Funct Neurosurg 2017;95:117-124.

6. MarketsandMarkets. Neuromodulation Market by Technology (Deep Brain Stimulation, Spinal Cord Stimulation, Transcranial Magnetic Stimulation), by Application (Depression, Parkinson's, Tinnitus, Alzheimer's, Epilepsy, Ischemia, Obesity) - Trends \& Global Forecast to 2020. 2015. www.marketsandmarkets.com/PressReleases/ neurostimulation-devices.asp. (Last accessed May 27, 2018).

7. Halpern CH, Mitchell GW, Paul A, et al. Self-administered preoperative antiseptic wash to prevent infection after deep brain stimulation. Am J Infect Control 2012;40:431-433.

8. Miller JP, Acar F, Burchiel KJ. Significant reduction in stereotactic and functional neurosurgical hardware infection after local neomycin/polymyxin application. J Neurosurg 2009;110:247-250.

9. Alves RV, Godoy R. Topical antibiotics and neurosurgery: Have we forgotten to study it? Surg Neurol Int 2010;1:1-22.

10. Bakhsheshian J, Dahdaleh NS, Lam SK, et al. The use of vancomycin powder in modern spine surgery: Systematic review and meta-analysis of the clinical evidence. World Neurosurg 2015;83:816-823.

11. Abdullah KG, Chen HI, Lucas TH. Safety of topical vancomycin powder in neurosurgery. Surg Neurol Int 2016; 7(Suppl 39):S919-S926.

12. Ravikumar V, Ho AL, Pendhakar AV, et al. The use of vancomycin powder for surgical prophylaxis following craniotomy. Neurosurgery 2017;80:754-758.

13. Amrani J. Intraoperative powdered vancomycin use with paddle lead placement. Neuromodulation 2015;18:177-181.

14. Rasouli JJ, Kopell BH. The adjunctive use of vancomycin powder appears safe and may reduce the incidence of surgical-site infections after deep brain stimulation surgery. World Neurosurg 2016;95:9-13.

15. Kang DG, Holekamp TF, Wagner SC, Lehman RA Jr. Intrasite vancomycin powder for the prevention of surgical site infection in spine surgery: A systematic literature review. Spine J 2015;15:762-770.

16. Kolek MJ, Dresen WF, Wells QS, Ellis CR. Use of antibacterial envelope is associated with reduced cardiac implantable electronic device infections in high-risk patients. Pacubg Clin Electrophysiol 2013;36:354-361.

17. Mittal S, Shaw RE, Michel K, et al. Cardiac implantable electronic device infections: Incidence, risk factors, and the effect of the AigisRx antibacterial envelope. Heart Rhythm 2014;11:595-601.

18. Peerdeman SM, Groot VD, Feller RE. In situ treatment of an infected intrathecal baclofen pump implant with gentamicin-impregnated collagen fleece. J Neurosurg 2010; 112:1308-1310.

19. Atiyeh BS, Hayek SN, Skaf GS, et al. Baclofen pump pocket infection: A case report of successful salvage with muscle flap. Int Wound J 2006;3:23-28. 
20. Aristedis R, Dimitrios P, Nikolaos P, Alexandros B. Intrathecal baclofen pump infection treated by adjunct intrareservoir teicoplanin installation. Surg Neurol Int 2017;8:38.

21. Bennett MI, Tai YM, Symonds JM. Staphylococcal meningitis following Synchromed intrathecal pump implant: A case report. Pain 1994;56:243-244.

22. Penn RD. Intrathecal baclofen for spasticity of spinal origin: Seven years of experience. J Neurosurg 1992;77:236-240.

23. Kallweit U, Harzheim M, Marklein G, et al. Successful treatment of methicillin-resistant Staphylococcus aureus meningitis using linezolid without removal of intrathecal infusion pump. Case report. J Neurosurg 2007;107:651-653.

24. Zed PJ, Stiver HG, Devonshire V, et al. Continuous intrathecal pump infusion of baclofen with antibiotic drugs for treatment of pump-associated meningitis. Case report. J Neurosurg 2000;92:347-349.

25. Yazdi C, Finn R. Management of intrathecal pump site infection in a patient with metastatic breast cancer without the removal of the system, a case report. J Anesth Int Care Med 2017;1:555-568.

26. Hester SM, Fisher JF, Lee MR, et al. Evaluation of salvage techniques for infection baclofen pumps in pediatric patients with cerebral palsy. J Neurosurg Pediatr 2012;10: 548-554.

27. Mekhail NA, Mathews M, Nageeb F, et al. Retrospective review of 707 cases of spinal cord stimulation: Indications and complications. Pain Pract 2011;11:148-153.
28. Wolfson A, Dlugy C, Shotland Y. Glycerol as a green solvent for high product yields and selectivities. Environ Chem Lett 2007;5:67-71.

29. Xu-Hui W, Chun Z, Guang-Jian S, et al. Long-term outcomes of percutaneous retrogasserian glycerol rhizotomy in 3370 patients with trigeminal neuralgia. Turk Neurosurg 2011;21:48-52.

30. Power WJ, Collum LM, Easty DL, et al. Evaluation of efficacy and safety of ciprofloxacin ophthalmic solution versus chloramphenicol. Eur J Ophthalmol 1993;3:77-82.

31. Saul JM, Ellenburg MD, de Guzman RC, Van Dyke M. Keratin hydrogels support the sustained release of bioactive ciprofloxacin. J Biomed Mater Res A 2011;98:544-553.

32. Unal S, Flokowitsch J, Mullen DL, Preston DA, Nicas TI. In-vitro synergy and mechanism of interaction between vancomycin and ciprofloxacin against enterococcal isolates. J Antimicrob Chemother 1993;31:711-723.

Address correspondence to: Dr. Christopher Payne Department of Neurosurgery Allegheny General Hospital 420 East North Avenue Pittsburgh, PA 15212-4756 USA

E-mail: paynech@tcd.ie 